
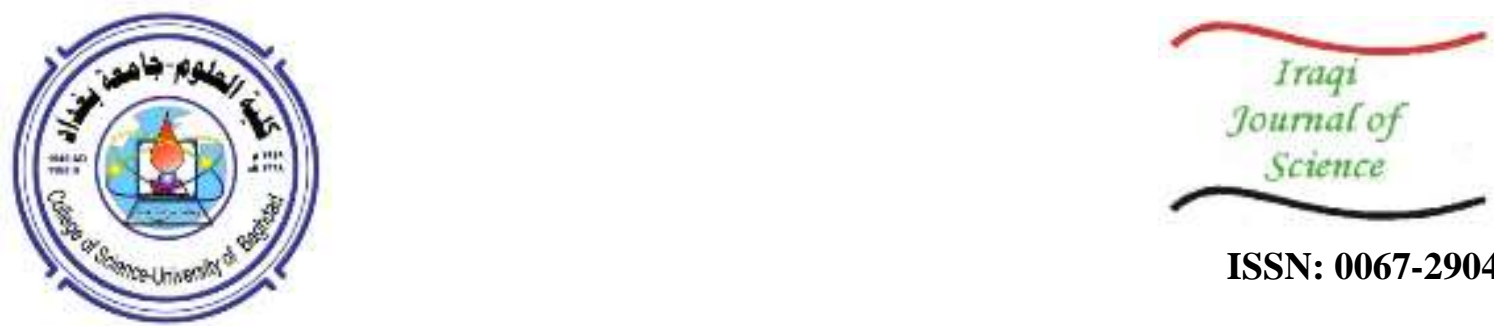

ISSN: 0067-2904

\title{
Enhancement of Wheat Leaf Images Using Fuzzy-Logic Based Histogram Equalization to Recognize Diseases
}

\author{
Fatima I. Abbas ${ }^{1}$, Nabeel M. Mirza ${ }^{1}$, Amel H. Abbas ${ }^{2}$, Layla H. Abbas ${ }^{2}$
}

${ }^{1}$ Department of Physics, College of Education, University of Mustansiriyah, Baghdad, Iraq

${ }^{2}$ Department of Computer Science, College of Science, University of Mustansiriyah, Baghdad, Iraq

Received: $17 / 11 / 2019 \quad$ Accepted: 19/4/2020

\begin{abstract}
The detection of diseases affecting wheat is very important as it relates to the issue of food security, which poses a serious threat to human life. Recently, farmers have heavily relied on modern systems and techniques for the control of the vast agricultural areas. Computer vision and data processing play a key role in detecting diseases that affect plants, depending on the images of their leaves. In this article, Fuzzy- logic based Histogram Equalization (FHE) is proposed to enhance the contrast of images. The fuzzy histogram is applied to divide the histograms into two subparts of histograms, based on the average value of the original image, then equalize them freely and independently to conserve the brightness of the image. The proposed method was evaluated using two well-known parameters: Mean Square Error (MSE) and Peak Signal to Noise Ratio (PSNR). The best results were reflected by MSE $=0.071$ and PSNR $=39.58$ for the Mildew Powdery disease. It is impressive to recognize that the proposed method yielded clear positive outcomes through producing better contrast enhancement while preserving the details of the original image, as confirmed by the subjective metrics.
\end{abstract}

Keywords: Wheat leaf diseases, Histogram equalization, Fuzzy-logic, Image quality measurement, contrast enhancement.

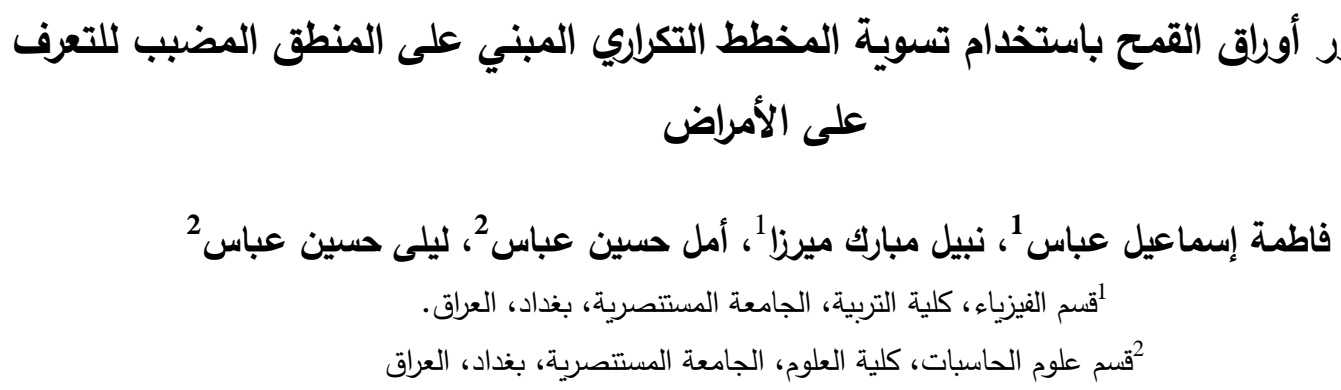

$$
\begin{aligned}
& \text { الخلاصة } \\
& \text { يعُُ إكثاف الأمراض التي تصيب النباتات بصورة عامة والقصح بصورة خاصة أمراً في غاية الأهمية }
\end{aligned}
$$

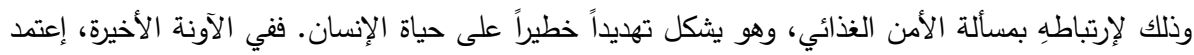

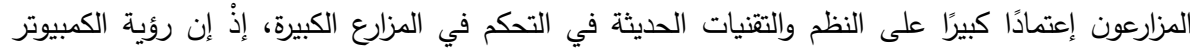

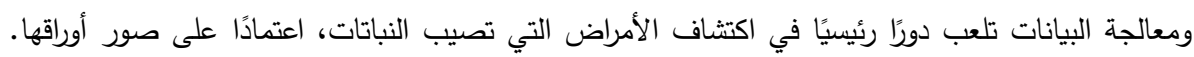

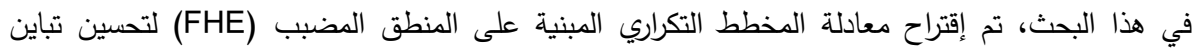

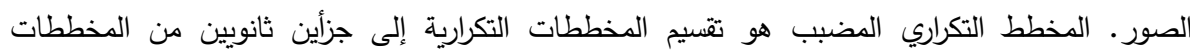

*Email: fatma11113333@yahoo.com 


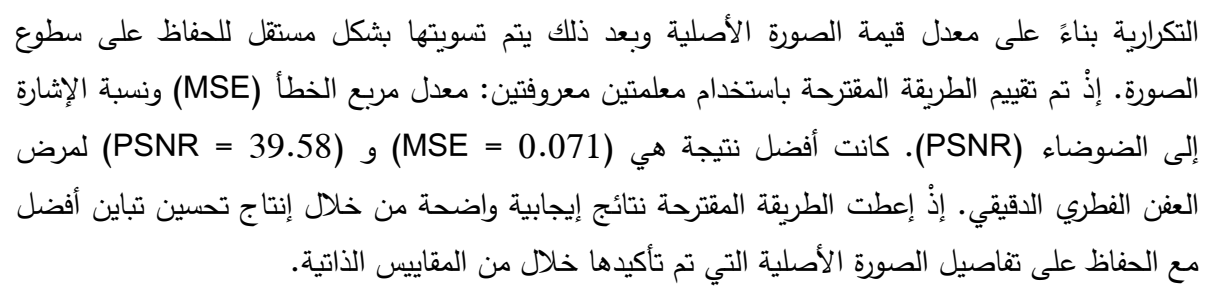

\section{Introduction}

Wheat in Iraq is a main food for all layers of the society, where grains are used for the production of bread and pastry. Wheat cultivation occupies strategic importance given to capacity trade-offs in the international market. Wheat is affected by various factors of biotic and non-biotic stress. Biotic stress includes that caused by fungi, bacteria and viruses, while non-biotic stress involves the effects of the surrounding environmental elements such as water, temperature, etc. There are various leaf diseases that affect wheat, which include leaf rust orange, yellow rust syndrome, and powdery mildew. Crop diseases reduce the yield significantly, sometimes to up to $50 \%$ or more. Measures to avoid disease and pest control in wheat plants play an important role in improving products. Human examination is the most common method for this task, but it is error-prone. Moreover, it is a way that leads to waste of time, effort and money. There is another challenge, where most wheat fields are located in rural areas, which requires farmers to travel long distances in order to communicate with experts. Image processing provides precision high speed, and time effectiveness, and does not require large expenditures. The best solution lies in the application of effective image processing techniques in detecting diseases affecting wheat, thus protecting food security [1].

Image processing techniques are used in several applications, such as underwater imaging, night vision, remote sensing, archaeology, medical imaging, etc. They are also utilized in plant disease diagnosis, where different techniques are used to identify the percentage of the affected areas. These include the enhancement, segmentation, thinning and filtration, among other techniques [2].

\section{Related Work}

Since a long time, one of the main concerns of researchers has been the diagnosis and control of diseases affecting leaves [3]. The following studies are cited to clarify the different methods for the purpose of enhancing images for plant disease diagnosis.

Zhang et al. [4] applied the histogram equalization method to enhance images as a preprocessing in their study that was intended to diagnose diseases affecting corn leaves. They reported that the recognition rate reached $87.8 \%$. El-Hely et al. [5] developed a special diagnostic system that relies mainly on image processing technology. Their system is a diagnostic model that they called as the Central Laboratory of Agricultural Expert System (CLASE) and used to manage cucumber crop as a case study. The CLASE observes the diseases on leaf image. They used four stages of image processing, including segmentation, enhancement, feature extraction and classification. They tested three different fungal disorders, which are Downey mildew, Powdery, and Leaf miner. The proposed system achieved a great success in the accuracy of diagnosis with a reduced error rate. Mousavi et al. [6] increased the contrast of the corn pest's images and then separated the affected areas by determining the threshold and means of the image's histogram. They were able to classify the spots of injury, with support from a vector machine classification system, with an average accuracy of $90.04 \%$. Al-Tarawneh [7] adopted the median filter and the conversion from RGB color model to $\mathrm{L}^{*} \mathrm{a}^{*} \mathrm{~b}^{*}$ color space for enhancing images of olive leaves. The researcher applied both of fuzzy c-mean classification (FCM) and k-mean classification (KMC) to determine the defected and affected areas in the leaves of plants. The accuracy rate achieved 66\% and $86 \%$ for KMC and FCM, respectively. Pengyun et al. [8] introduced a method to detect the spores automatically. They converted RGB images of plant disease (spores) to gray images, then used a median filter and canny edge algorithm to enhance the edges, converted gray image into binary image for segmentation, and extracted the features of spores. Rothe and Kshirsagar [9] suggested a method for classifying and identifying three categories of diseases that affect cotton leaf (bacterial Blight, Myrothecium, and Alternaria). The researchers used the Snake segmentation method and Hu's moments to extract the features. They utilized seven moments for training and a back propagation neural network for classification, and achieved a classification accuracy of 85\%. Bikash et al. [10] proposed Neural Network Ensemble (NNE) to recognize tea leaf diseases. Their approach relied on several pre-processing steps, such as image cropping, image 
resizing, and image converting to threshold value, reaching a recognition accuracy of $91 \%$. Abbas $e t$ al. [11] used the segmentation methodology to determine the percentage of affected areas in maize leaves. Their methodology relied on image classification by K-Means clustering along with image segmentation using Color Threshold, and then estimating the affected area by calculating the number of white pixels and dividing it by the number of total pixels.

\section{Wheat Diseases Analysis and Symptoms}

In this section, we describe the common infections that influence wheat and their symptoms.

\section{Leaf Rust}

The main attribute of leaf rust contagions is small orange structure sores [12]. These contagions are most common on leaves; it appears on the cover of the leaf and expands from the bottom of the leaf surface to the stem node. Lesions induced by leaf corrosion are usually smaller and rounder and cause less tearing of the leaf structure than those triggered by stem rust [12].

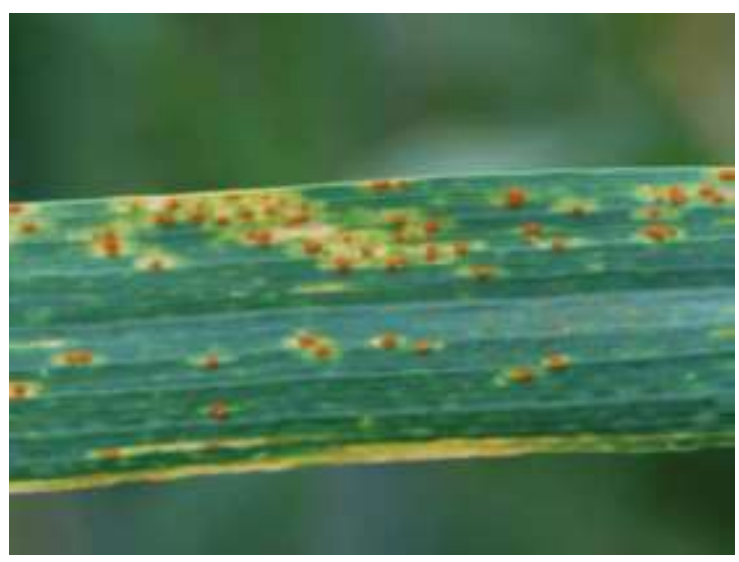

Figure1-Leaf Rust [12]

\section{Powdery Mildew}

Indications of powdery mildew include white contagious outgrowths on leaves and leaf sheaths (Figure-2). It does not stop there, but glumes and awns will also be covered by infections, depending on the seriousness of the disease. The infection growth is often confined to the outer surfaces of plants and it can be effectively cleaned by touching the affected area with fingers. Fungal growth lesions may be dark brown or blended with white fungus growth (cottony) [12].

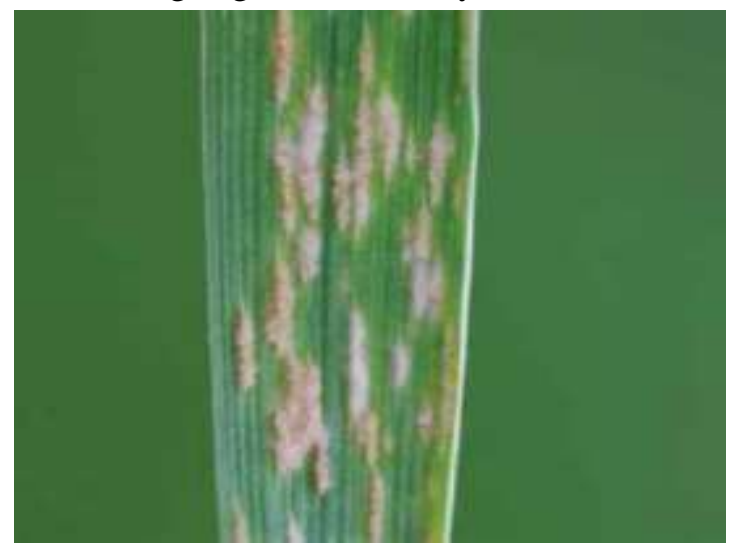

\section{Tan Spot}

Figure 2-Powdery Mildew [12].

The side symptoms of tan spot are small dark colored spots that grow to wind up tan curve-shaped or elliptical lesions with a yellow surrounding (Figure-3). Often, there is a small, dark brown spot in the center of the lesion [13]. The injuries frequently converge as they develop, bringing about expansive segments of influenced tissue. Eventually, the old leaves begin to die. 


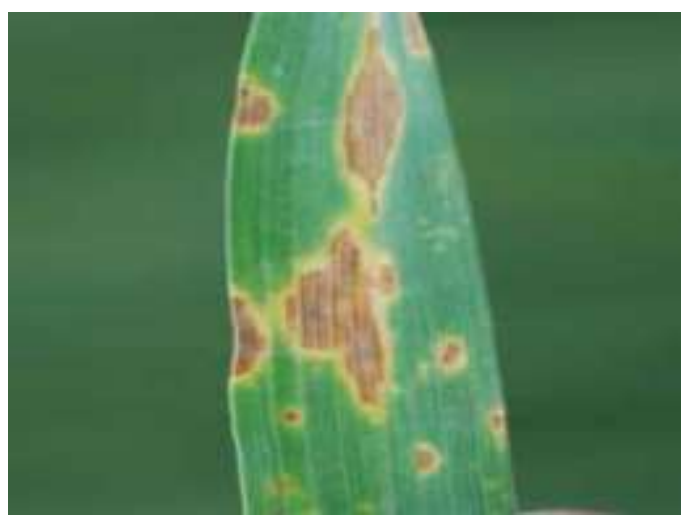

Figure 3- Tan Spot [12].

\section{The Proposed Method}

This paper proposes Wheat Leaf Disease Image Enhancement (WLDIE), which used to examine the effects of diseases on wheat leaves. The information of WLDIE based on the images of wheat leaves taken by a digital camera. WLDIE is consisting of four phases: image acquisition, preprocessing, enhancement, and then quality measurement. Figure-4 represents the scheme for the proposed method.

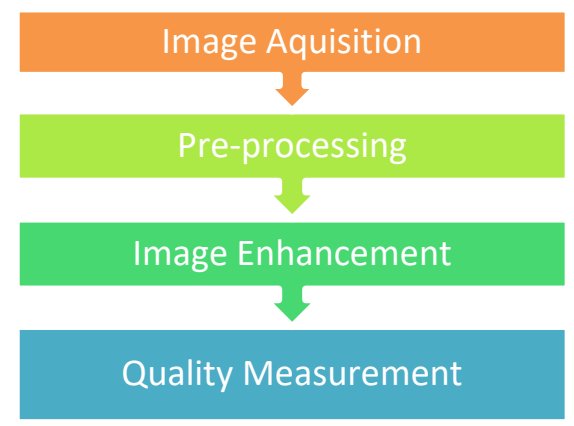

Figure 4-Scheme of the proposed method.

\section{Image Acquisition}

Making images for plant leaves was achieved by capturing pictures using a digital camera with appropriate resolution for that scene and then saving them in RGB format. Three common types of wheat leaf diseases were selected from the international maize and wheat organization website (CIMMYT) [12], which included Leaf Rust, Powdery Mildew, and Tan Spot.

\section{Pre-processing}

To remove unwanted parts, noise from images and improve their quality, two procedures adopted including:

\section{Image Cropping}

The image that captured through a digital camera contains about $30 \%$ of the infected plant leaf information. The remaining $70 \%$ of the information is not important because it represents the background that occupies a volume of memory space, it also effects on the desired results of this operation. In order to provide space in the storage and speed in processing, it is imperative to crop the uninteresting regions of the image. There are two common methods that can be used to crop the image. The first is by using the command imcrop (I) that uses the interactive cropping tool, where (I) is the inserted image which needs to be cropped. This method provides a high degree of flexibility and allows the user to crop any required part of the image, as shown in Fig. 5. Therefore, this method recommended because it is more flexible for the user and there will be no need to worry about losing any important information in the image that may be necessary to detect the disease. 


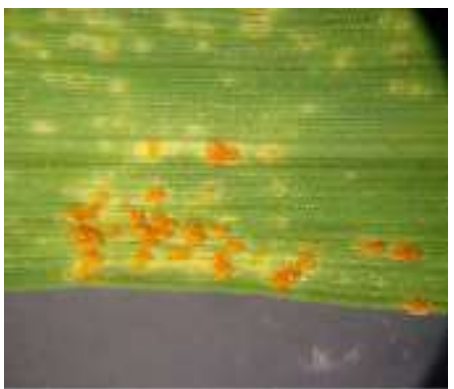

(a)

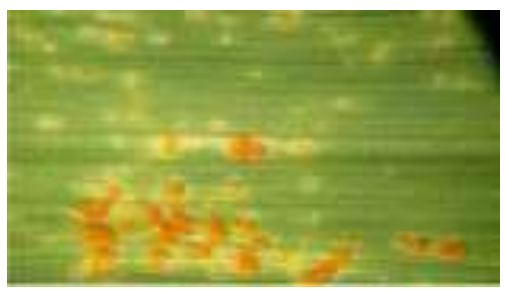

(b)

Figure 5-(a) Original image and (b) Image after cropping by the first method.

The second method relies on specifying the area points to be cropped, that contain the coordinates $\mathrm{x}_{1}, \mathrm{x}_{2}, \mathrm{y}_{1}$, and $\mathrm{y}_{2}$. This method depends on the pre-selection of the unwanted part by determining the coordinates of those parts, where a cropping operation cannot be edited without the object's coordinates. Thus, this method is not useful, mostly because it requires that all images be $100 \%$ of equal size.

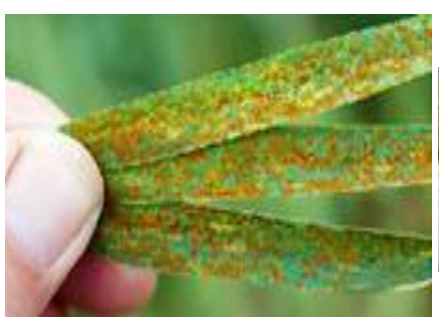

(a)

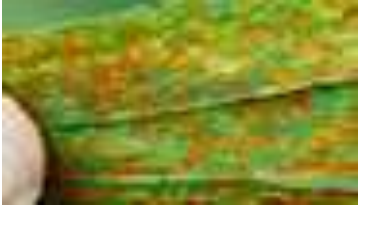

(b)

Figure 6- (a) Original image and (b) Image after cropping by the second method.

\section{Image Denoising}

Image denoising is the way to reconstruct an original image and remove the noise from it. Removing noise is a prerequisite operation in the image-processing field. It is known that the median filter has the ability to remove the noise type of "salt and pepper". In addition, it is considered as the basis for the most advanced image filtering applications, such as pattern recognition, object segmentation, and medical imaging. Therefore, in this work, the median filter was adopted for removing noise.

\section{Image Enhancement}

The phase of image improvement is one of the most important proceedings for specialists in many of the applied fields, because it contributes to the diagnosis of the accurate contents and details of the image. Therefore, wheat disease images were improved using three different methods.

\section{Histogram Equalization}

Histogram Equalization (HE) is a simplest and easiest histogram modelling technique known. In the HE method, the resultant image is often flat or nearly flat, i.e. it contains a uniform distribution of intensity. In other words, the intensity of the image becomes more uniform, with better contrast and more detailed image of bright or dark regions. Thus, the contrast of the resulted image is enhanced. The histogram equalization formula is expressed in equation (1) while its working steps are shown in algorithm 1 .

$$
H(g)=\left(\frac{C D F(g)-C D F(g)_{\min }}{M \times N} \times(255)\right)
$$

where $C D F_{\min }$ represents the minimum non-zero value of the Cumulative Distribution Function $(\mathrm{CDF})$, and $(\mathrm{M} \times \mathrm{N})$ represents the size of image that has a width of $\mathrm{M}$ and a height of $\mathrm{N}$.

\section{Fuzzy Histogram Equalization}

Fuzzy histogram equalization (FHE) is proposed to enhance the contrast of images. FHE consists of two procedures; firstly, computing a fuzzy histogram depending on the set theory to address the gray level values to the best. Secondly, dividing the fuzzy histogram found in the first procedure into two 
sub histograms based on the median value of the initial image. Then each sub histogram is equalized independently in order to maintain the brightness of the image. A fuzzy histogram is defined as a set of real numbers that are sequenced as $\boldsymbol{f}(\mathrm{g})$ and $g \in\{0,1, \ldots, 255\}$,

where $\boldsymbol{f}(\boldsymbol{g})$ represents the recurrence of gray levels that has a value of $(\boldsymbol{g})$. If the gray value $I(r, c)$ is replaced as a fuzzy real number $\tilde{I}(r, c)$, then FHE can be computed by equation (2) and its working steps are described in algorithm 2:

$$
f(g) \leftarrow h(g)+\sum_{r} \sum_{c} \mu_{\tilde{I}(r, c) g}, k \in[a, b]
$$

where $\mu_{\tilde{I}(r, c) g}$ represents the triangular fuzzy membership function defined as:

$$
\mu_{\tilde{I}(r, c) g}=\max \left(0.1-\frac{I(r, c)-g}{4}\right)
$$

and $[a, b]$ is the support of the membership function.

\section{Contrast Stretching}

Contrast Stretching (CS) is a simple technique for enhancing images. It is a piecewise linear function that increases the dynamic range of the gray levels. The principal goal of improvement is to process the color image so that the result is more favourable than the initial image for any application. Stretching contrast can be calculated as in equation (4) and its working steps are Illustrated in algorithm 3:

$$
\operatorname{Stretch}(I(r, c))=\left[\frac{I(r, c)-I(r, c)_{\min }}{I(r, c)_{\max }-I(r, c)_{\min }}\right] \times[M A X-M I N]+M I N
$$

where $I(r, c)$ potential is the major grey- level in the image $I(r, c)$, and $I(r, c)_{\min }$ is the tiniest greylevel in the image $I(r, c)$. MAX and MIN indicate the maximum and minimum possible gray level values for an 8-bit grayscale image, which are usually 0and 255 , respectively.

\section{Image Quality Measurement}

For destroyed images that are damaged due to a certain type of noise, there is no simple quantitative measure for removing noise optimally. Certainly, the resultant image after the filtration process is not perfect. Therefore, the overall visual quality must be tested. MSE gives an indication of the performance of the filter operation $[14,15]$. The resulting image error can be determined by the difference between the original and reconstructed pixel values, as follows:

$$
\operatorname{Error}(r, c)=x(r, c)-\hat{x}(r, c)
$$

where $x(r, c)$ and $\hat{x}(r, c)$ represent the original and reconstructed pixel values, respectively. After that, the total error between the original image and the reconstructed image to size $(\mathrm{M} \times \mathrm{N})$ can be determined as:

$$
\text { Total Error }=\sum_{r=0}^{M-1} \sum_{c=0}^{N-1}(x(r, c)-\hat{x}(r, c))
$$

The MSE is ready by taking the total of the squared differences divided by the size of image, as in the following:

$$
M S E=\frac{1}{M \times N} \sum_{r=0}^{M-1} \sum_{c=0}^{N-1}(x(r, c)-\hat{x}(r, c))^{2}
$$

PSNR is a common and important method used to measure error ratio. It is inversely proportional to the value of MSE and is written as follows [14, 15]:

$$
P S N R=10 \times \log 10\left(\frac{255^{2}}{M S E}\right)
$$




\section{Algorithm (1)}

Input: Insert images of the wheat disease.

Output: Image enhancement by histogram equalization. Algorithm steps:

(a) Input color image $(I(r, c))$ then crop using imcrop command.

(b) Normalize image: $r_{j}(g)=I_{g}(r, c) / 255$.

(c) Compute histogram: $P\left(r_{j}(g)\right)=n_{j}(g) / N$.

where: $\mathrm{j}=0,1, \ldots 255$. $\left(n_{g}\right)$ is the number of pixels of gray level $\left(r_{g}\right)$, and $(\mathrm{N})$ is the size of image.

(d)Calculate cumulative histogram by (eq. 9):

$$
S_{k}(g)=\sum_{k=0}^{255} \frac{n_{k}(g)}{N}
$$

\section{Algorithm (2)}

Input: Insert images of the wheat disease.

Output: Image enhancement by fuzzy histogram equalization. Algorithm steps:
(a) Input color image then crop using imcrop command.
(b) Apply median filter to remove noise.
(c) Convert the gray level intensity of image to fuzzy plane ( 0 and 1$)$ which indicates (dark and bright).
(d) Reduce the rate of fuzzy using contrast enhancement (eq. 3).
(e) Compute fuzzy histogram using (eq. 2).

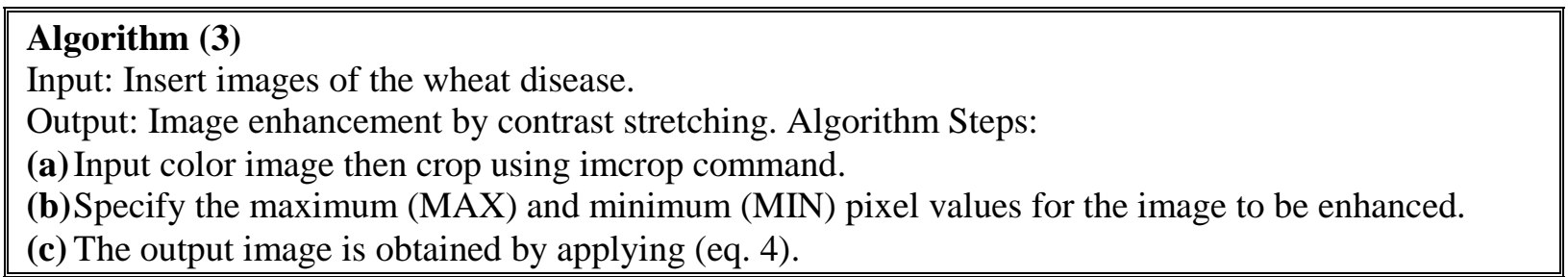

\section{Results and Discussion}

The study is focused on images of three types of wheat diseases, two images for each type. The first row of Figure- 7 shows the original images, while the second row of shows the original images after being cropped.

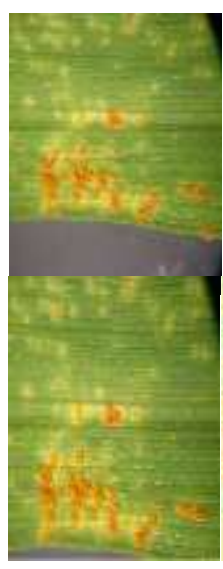

a1

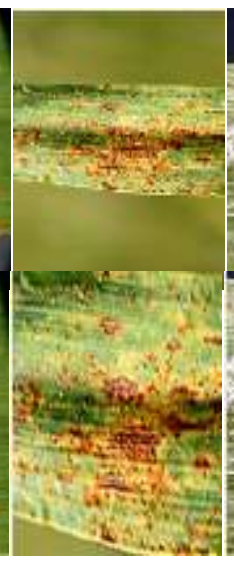

a2

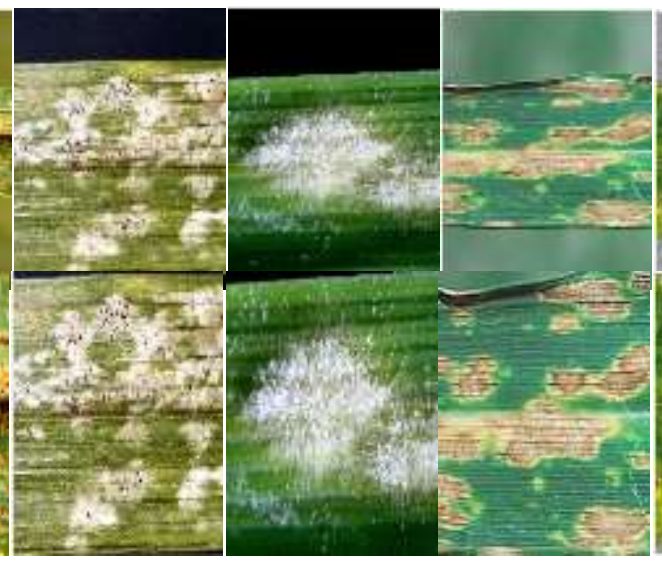

b1 b2

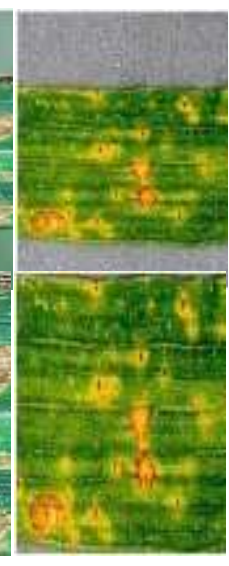

c2

Figure 7-Original images for diseases: (a) Orange Rust, (b) Powdery Mildew, and (c) Tan Spot.

Figure-8 illustrates a comparison between traditional methods of enhancement, including HE and CS and the enhancement based on the fuzzy concept (FHE). With regard to the effects of color and intensity, it appears visually from Figure 8- first row that the FHE method has produced improved images of better quality than other enhancement methods. 


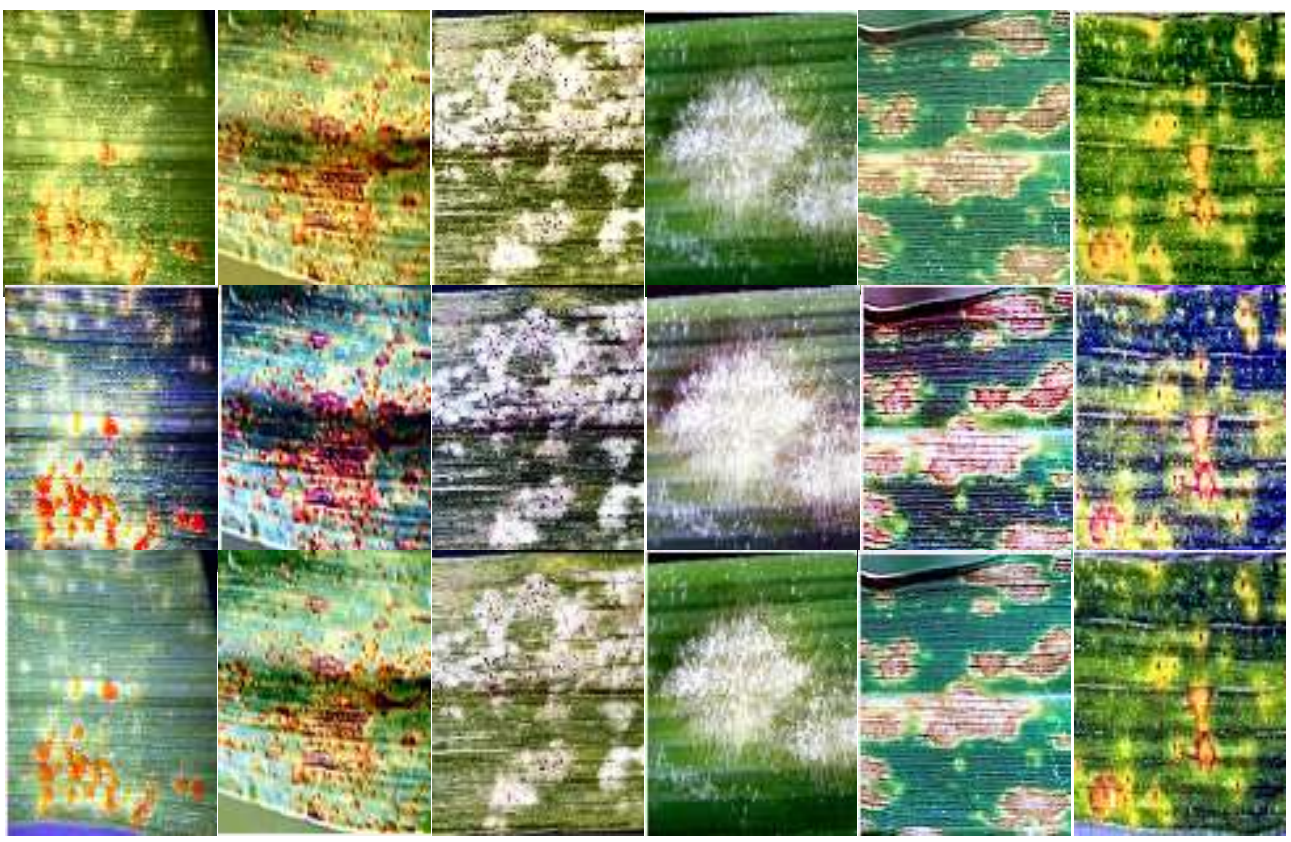

Figure 8-Results of images enhancement. First row: Images enhanced by FHE, second row: Images enhanced by HE, and third row: Images enhanced by CS.

In this paper, the methods of enhancement were tested by the subjective metrics assessment depending on the values of mean squared error and peak signal-to-noise ratio. As is well known, MSE measures the difference between two samples, such as original - enhanced or original - distorted, etc. According to earlier reports [16, 17], whenever this value is near zero, the model is close to the ideal state and vice versa. The exact is opposite in relation to PSNR, as it is inversely proportional to MSE. While, typical values of PSNR are between 30 and $50 \mathrm{~dB}$ when image elements are 8-bit. In other words, the higher value indicates the best level $[17,18]$.

From Tables- $(1,2)$, it is clear that the fuzzy histogram equalization method resulted in very good images for Orange Rust a2 and Mildew Powdery b2 diseases, where it was observed that the PSNR values were within the range of typical values, as marked with bold font $(30.4005,39.5861$ and 32.9116) in Table- 1. While, the histogram equalization showed undesired results and the contrast stretching method resulted in acceptable outcomes. In the same context, the metric of quality evaluation (MSE) also proved the efficiency of the proposed method, based on its values which are marked by the bold font in Table-2.

As for Tan spot disease images, the results were similar using both fuzzy histogram equalization and contrast stretching, with a simple preference for the first method. Whereas, the histogram equalization method produced the worst results. Although the results of the images of Mildew disease are not much different from the previous ones, they are slightly better for the fuzzy histogram equalization, whereas the histogram equalization remained of reduced quality.

Table 1-Subjective assessment (PSNR values) and comparison among methods of enhancement

\begin{tabular}{|c|c|c|c|}
\hline Images & PSNR $_{\text {FHE }}$ & PSNR $_{\text {HE }}$ & PSNR $_{\text {CS }}$ \\
\hline Orange Rust (a1) & 18.6493 & 11.8463 & 15.2875 \\
\hline Orange Rust (a2) & $\mathbf{3 0 . 4 0 0 5}$ & 13.8589 & 19.08 \\
\hline Mildew Powdery (b1) & 22.4701 & 16.6655 & 21.9313 \\
\hline Mildew Powdery (b2) & $\mathbf{3 9 . 5 8 6 1}$ & 15.4143 & $\mathbf{3 2 . 9 1 1 6}$ \\
\hline Tan Spot (c1) & 28.5092 & 15.8916 & 21.8148 \\
\hline Tan Spot (c2) & 19.6994 & 10.1856 & 16.837 \\
\hline
\end{tabular}


Table 2-Subjective assessment (MSE values) and comparison among methods of enhancement

\begin{tabular}{|c|c|c|c|}
\hline Methods & MSE $_{\text {FHE }}$ & MSE $_{\text {HE }}$ & MSE $_{\text {CS }}$ \\
\hline Orange Rust (a1) & 8.87 & 42.5 & 19.24 \\
\hline Orange Rust (a2) & $\mathbf{0 . 5 9}$ & 26.7 & 8.03 \\
\hline Mildew Powdery (b1) & 3.68 & 14.1 & 4.16 \\
\hline Mildew Powdery (b2) & $\mathbf{0 . 0 7 1}$ & 18.7 & $\mathbf{0 . 3 3}$ \\
\hline Tan Spot (c1) & 0.916 & 16.7 & 4.28 \\
\hline Tan Spot (c2) & 6.97 & 62.3 & 13.47 \\
\hline
\end{tabular}

\section{Conclusions}

Pre-processing is a necessary process to optimize the image to be ready for analysis and optimization and thus obtain the suitable results. The cropping process is an important procedure to reduce the unnecessary information, thus saving both memory space and time for processing. From the subjective and objective measures, it was clear that the Fuzzy Histogram Equalization (FHE) is the best method in most cases; the Contrast Stretching (CS) gave acceptable results, while Histogram Equalization (HE) was the worst method.

\section{References}

1. Flood, J. 2010. "The Importance of Plant Health to Food Security", Food Security, 2(3): 215-231, DOI: $10.1007 / \mathrm{s} 12571-010-0072-5$.

2. Sumana, S., Sakhamuri, S. and Sataya, V.B.B. 2019. "An Efficient Classification Model for Plant Disease Detection", International Journal of Innovative Technology and Exploring Engineering (IJITEE), 8(7): 126-129.

3. Pujari, J., Yakkundimath, R. and Byadgi, A. 2016. "SVM and ANN Based Classification of Plant Diseases Using Feature Reduction Technique", International Journal of Interactive Multimedia and Artificial Intelligence, 3(7): 6-14.

4. Zhang, Z., Li, Y., Wang, F. and He, X. 2014. "A Particle Swarm Optimization Algorithm for Neural Networks in Recognition of Maize Leaf Diseases", Sensors \& Transducers, 166(3): 181189.

5. El-Helly, M., Rafea, A., El-Gamal, S. and Abd El Whab, R. 2004. "Integrating Diagnostic Expert System with Image Processing Via Loosely Coupled Technique", Central Laboratory for Agricultural Expert System (CLAES), Proceedings of $2^{\text {nd }}$ International Conference on Informatics and Systems, pp. 143-149.

6. Mousavi, S., Hanifeloo, Z., Sumari, P. and M. Arshad, M. 2016. " Enhancing the Diagnosis of Corn Pests Gabor Wavelet features and SVM Classification", Journal of Scientific \& Industrial Research, 75: 349-354.

7. Mokhled Al-Tarawneh, S. 2013. "An Empirical Investigation of Olive Leave Spot Disease Using Auto-Cropping Segmentation and Fuzzy C-Means Classification", World Applied Sciences Journal, 23(9): 1207-1211.

8. Pengyun, X. and Jigang, L. 2009. "Computer Assistance Image Processing Spores Counting", International Asia Conference on Informatics in Control, Automation and Robotics, 2009 IEEE computer society, pp. 203-206.

9. Rothe, R.R. and Kshirsagar, R.V. 2015. "Cotton Leaf Disease Identification Using Pattern Recognition Techniques", International Conference on Pervasive Computing (ICPC), 2: 1-6.

10. Karmokar, C., Ullah, M.S., Siddiquee, M. and Alam, K.R. 2015. "Tea Leaf Diseases Recognition Using Neural Network Ensemble", International Journal of Computer Applications, 114(17): 2730.

11. Abbas, A., N. Mirza, Qassir, S. and Abbas, L. 2020. "Maize Leaf Images Segmentation Using Color Threshold and K-means Clustering Methods to Identify the Percentage of the Affected Areas", IOP Conf. Ser.: Mater. Sci. Eng. 745012048.

12. The CIMMYT Maize Program. 2004. "Maize Diseases: A Guide for Field Identification", $4^{\text {th }}$ edition Mexico, D. F.: CIMMYT. 
13. Bhavani, S. and Diana, I. 2018. "Image Segmentation: Using K-Means Algorithm and Threshold Method", International Conference on Advancements in Computing Technologies - ICACT, February 2018, 4(2): 5-8.

14. Al-Dabbas, H.M. and Morad, A.H. 2019. "Medical Image Enhancement to Extract Brain Tumors from CT and MRI images", Iraqi Journal of Science, 60(8): 1820-1829.

15. Habeeb, N.J. 2019. "Comparative analysis of Median filter family for Removing High-Density Noise in Magnetic Resonance Images", Iraqi Journal of Science, 60(10): 2246-2256.

16. Rastogi, J., Mittal, D. and Singh, D. 2017. "Comparative Study of Image Denoising Algorithms in Medical and Satellite Images", International Research Journal of Engineering and Technology (IRJET), 04(04) Apr.

17. Stephen, T. and Welstead, 1999. "Fractal and wavelet image compression techniques", SPIE Publication. pp. 155-156.

18. Mauro Barni, 2006. "Fractal Image Compression", Document and Image Compression (CRC Press), $1^{\text {st }}$ Edition. 